\title{
Traumatismo dental en bebés
}

Antonio Jose da Silva Nogueira ;

Rogério da Gama Malcher Nogueira²;

Daniela Barros Corrêa ${ }^{3}$;

Flávia Xavier Souza ;

\section{Resumen}

Las ocurrencias traumáticas pueden traer consecuencias desagradables tanto en lo físico como en lo estético, como en el aspecto psicológico. Los traumas sufridos por bebés traen situaciones casi irreparables en el momento, tales como las limitaciones inherentes a la edad del paciente cuando aún es bebé. Las soluciones protésicas, por ejemplo, son casi siempre descartadas porque la edad del paciente es incompatible con el uso de una prótesis. Así, como ésta, también ocurren muchas otras situaciones, limitando un tratamiento inmediato y representando para el paciente un problema a ser resuelto. Por lo tanto, en el trabajo se hace una revisión de la bibliografía que aborda el traumatismo dental en bebés y comentarios sobre los exámenes a realizar, siempre considerando la edad, crecimiento y desarrollo, además de relatar las consecuencias de esos traumatismos en esta franja etaria inicial de vida como resultado. Finalmente, trata de alertar a los responsables de los niños de los cuidados de emergencia que se deben tener en algunas ocurrencias de traumatismos dentales en bebés.

Palabras clave: Traumatismo, dentición, niño.

Artigo de Revisão

\section{Traumatismo dental em bebês}

\section{Resumo}

As ocorrências traumáticas podem trazer consequências desagradáveis seja na questão física como na estética, ou no aspecto psicológico. Os traumas acometidos em bebês, trazem, situa- ções quase que irreparáveis no momento, tais como as limitações inerentes à idade do paciente quando ainda bebê. As resoluções protéticas, por exemplo, são quase sempre descartadas, uma vez que a idade do paciente é incompatível com o uso de uma prótese. Assim, como esta,

\footnotetext{
1. Profesor titular, Doctor de Odontopediatría y Profesor en la Universidad Federal de Pará, Belén, Brasil.

2. Maestro en Implantodoncia por la Facultad de Odontología São Leopoldo Mandic, São Paulo, Brasil.

3. Cirujano Dentista formado por la Escuela Superior de la Amazonía, Manaos, Brasil.

4. Cirujano Dentista formado por la Escuela Superior de la Amazonía, Manaos, Brasil.
} 
muitas outras situações também ocorrem, limitando um tratamento imediato, representando um problema a ser resolvido para o paciente. Assim sendo, no trabalho é feito uma revisão de literatura que aborda o traumatismo dentário em bebês e comentários sobre os exames a serem feitos, sempre considerando a idade, crescimento e desenvolvimento, além de relatar as consequências desses traumatismos nesta faixa etária inicial de vida como resultado. Por fim, trata de alertar os responsáveis pelas crianças, dos cuidados emergenciais que se deve ter em algumas ocorrências de traumatismos dentais em bebês.

Palavras chaves: Traumatismo, dentição, criança.

Review Article

\section{Dental trauma in infants}

\begin{abstract}
Traumatic events can result in unpleasant outcomes, both physically and aesthetically, including psychological aspects. Dental trauma in infants results in almost irreparable damages at the time, such as limitations inherent to the patient's age. For instance, prosthetic interventions for infants are almost always disregarded as the patient's age is not compatible with the use of prosthesis. Therefore, many other similar situations involving infants may also occur, which
\end{abstract}

\section{Introducción}

Los traumas bucofaciales y dentales se presentan, casi siempre, en algún momento de la infancia, así como se hacen presentes los descubrimientos de circunstancias peligrosas donde los niños, sin suficiente madurez psicológica, quienes pasan por situaciones perjudiciales que pueden generar un trauma bucodental.

Los problemas traumáticos relacionados con la boca y los dientes han sido tratados frecuentemen- limits any immediate treatment and represents a problem to be solved. The present work aims to review the literature on dental trauma in infants and discuss on the exams to be performed, always considering age, growth and development of the patient, in addition to reporting traumatic outcomes in this early age group. Finally, the present work also attempts to advise the caregivers about the emergency care they should follow in some occurrences of dental trauma in infants.

Keywords: Trauma, dentition, child.

te en la literatura mundial bajo los más diversos temas como, por ejemplo, sus etiologías, consecuencias, formas, afección de otros órganos y, no menos importante, bajo los efectos psicológicos que se suceden en los niños y en sus acompañantes o responsables.

En la literatura, se observa la preocupación con qué hacer en las ocurrencias traumáticas o cuáles son sus efectos para las denticiones. Son frecuentes algunas preguntas como: ¿Cuáles son los tipos de tratamientos en el caso de los niños en edades tem- 
pranas y autolimitantes? ¿Qué se puede hacer para suplir esa dificultad? ¿Cuándo podremos intervenir y cómo, a fin de reducir los efectos indeseables para el propio crecimiento bucofacial del niño? Estas son interrogantes frente a los traumas bucodentales en niños de poca edad que necesitan de mayor atención.

Por ello, para lograr nuestro objetivo, queremos abrir fuentes de estudios y explicaciones que puedan minimizar los efectos negativos para el desarrollo normal y secuencial del niño.

\section{Revisión de Literatura:}

Según Baldini y Cols. ${ }^{1}$ las características de la boca de un bebé son únicas y particulares a este período de la vida. La boca, así como todo el resto del cuerpo, está en constante desarrollo.

Corrêa ${ }^{2}$ afirma que los profesionales que tratan y acompañan a los niños por toda su niñez e infancia, serían privilegiados en detectar primero algún tipo de anomalía. La Odontología en bebés enfatiza la atención precoz y crea un escenario muy favorable a esta detección de situaciones anormales en la cavidad oral del niño. Sin embargo, es necesario que se ofrezca cada vez más este servicio a la población, ya que muchos niños con lesiones dentales no son tratados inmediatamente, lo que puede ocurrir por la falta de acceso a la atención dental.,

Asunção y Ferelle y Oliveira $^{6}$ apuntan innumerables consecuencias para las denticiones causadas por los traumatismos. La decoloración coronaria es uno de los tipos de secuelas más notadas, incluso por autores como Borum y Andreasen, ${ }^{7}$ Holan y Ram, ${ }^{8}$ Cardoso y Rocha, ${ }^{9}$ y Holan y Fuks. ${ }^{10}$ Para Malmgren y Cols. ${ }^{11}$ la decoloración blanca o amarillo-marrón de corona e hipoplasia de los incisi- vos permanentes son, no obstante, la secuela más común después de la intrusión y de la avulsión de los dientes deciduos en niños durante las edades de 1-3 años.

A pesar de la aparición de hipoplasia de esmalte en la dentición decidua, debido a la ocurrencia de traumatismo, Chagas y Cols. ${ }^{12}$ no identificaron asociación estadísticamente significativa entre esa anomalía y el traumatismo.

Pugliesi y colaboradores ${ }^{13}$ mencionan los traumas del tipo subluxación e intrusión como los más prevalentes entre los niños. Los factores como la edad del niño, cuando de la ocurrencia traumática, el grado de desplazamiento dental y la pérdida de estructura, pueden influir en las consecuencias presentadas. ${ }^{7}$

Las caídas son los factores etiológicos de mayor prevalencia. ${ }^{14,15}$ El lugar preferencial para la ocurrencia traumática dental en los niños de edad temprana, es el propio hogar. ${ }^{16}$

Zembruski-Jaber y Cols. ${ }^{17}$ y Souza y Cols. ${ }^{18}$ afirman que a pesar de los resultados en cuanto a la prevalencia de los traumas en niños ser altos, la prevención a estos eventos está fuera del control de los familiares, por tratarse de situaciones inesperadas y delcotidiano del niño.

Para Nogueira y Cols. ${ }^{19}$ y Granville-García y Cols. ${ }^{20}$, el grupo de edad inferior a cuatro años (4) es el más susceptible a los traumas bucodentales y la mayoría de estos niños no son llevados a la atención inmediata de emergencia. También se sugiere la aplicación de programas preventivos para los traumas dentales.

Valle y colaboradores ${ }^{21}$, estudiando la ocurrencia de lesiones traumáticas en niños de 0 a 36 meses, no 
observaron gran frecuencia de traumas (22,5\%), y las fracturas coronarias fueron las que más se sucedieron. Como etiología, la caída fue la más frecuente $(23,8 \%)$ y la observación y seguimiento periódico del paciente fue el tratamiento más empleado.

Con hallazgos equivalentes en cuanto a la etiología, Dorado y Cols. ${ }^{22}$ encontraron que las fracturas faciales tienen una prevalencia pequeña en niños menores de cinco (5) años. Los autores también evidenciaron que los niños están pasibles de agresiones físicas, y reiteraron que el mejor proceso es la prevención a los traumas, ya que, a pesar de las diversas opciones de tratamiento, ninguna de ellas trae resultados bastante satisfactorios. Es aceptado que los tratamientos son más difíciles, cuando los procesos de crecimiento y desarrollo están aún actuando sobre el niño.

\section{Principales causas de los traumatismos dentales en los bebés:}

Por la propia condición de dependencia, desconocer el peligro, no tener los movimientos locomotores bien desarrollados y las reacciones de defensa aún lentas según su razonamiento, los niños de hasta 2 o 3 años necesitan atención frecuente de sus responsables o acompañantes.

La etiología de los traumatismos en los bebés es variada en los procesos iniciales de vida, como ya hemos descrito y como si no bastara ese aprendizaje, a veces doloroso para caminar, el bebé estará predispuesto a caer del andador, del coche de bebé, del cambiador e incluso del cuello de sus responsables. En todas estas situaciones, la cabeza funcionará como un órgano prominente en el trauma y así, la presencia de dientes, aunque sean pocos, o incluso únicos, también puede agravar el caso y, a menudo nos deja sin alternativas terapéuticas, aunque momentáneamente, debido a la inmadurez del niño o de su dentición.

Los dientes intraóseos pueden ser alcanzados, bastando que el trauma incida directamente en la región de alvéolos, pudiendo causar alteración de la posición dental o incluso fractura de los elementos dentales, considerándose la intensidad del trauma.

Necesitamos estar conscientes de que correr, saltar, subir y las demás actividades serán rutinas para los niños y como tal, traerán gran cantidad de ocurrencias traumáticas. Ello porque, a pesar de haber el dominio de la locomoción, el instinto infantil de defensa en una caída, por ejemplo, todavía no es ágil, pues su proceso de maduración psicológica aún no es integral.

Accidentes de tránsito, agresiones a los niños, convulsiones, overjet exagerado o situaciones quirúrgicas donde hay necesidad de intubación del niño, son también causas frecuentes de traumas dentales. ${ }^{2}$

\section{Evaluación:}

A pesar de la limitación de edad, al bebé que sufre trauma bucodental y facial se le deben realizar radiografías para diagnosticar el problema, su extensión e intervenir lo más pronto posible, aunque con medidas parciales o incluso para alertar a los responsables para el seguimiento del caso.

Por consiguiente, podrá ser realizada radiografía periapical con la película infantil o la modificada, utilizando la película periapical adulta. Además, puede ser utilizada la radiografía extrabucal lateral, hecha con película oclusal en caso de bebés, proporcionando mayor comodidad a ellos. Es posible, 
recurrir a las radiografías extrabucales, hechas en clínicas radiológicas médicas, pues tal vez en ellas existan más condiciones de obtener mejores informaciones radiográficas.

Debe ser hecha una anamnesis objetiva con los acompañantes y atentar a la sospecha de pérdida de algún elemento dental, lo que necesita ser exhaustivamente investigado con los acompañantes que estuvieron presentes en el momento del acontecimiento traumático, para que se pueda alejar la sospecha de una aspiración del diente.

En caso de sangrado, hay que detenerlo y limpiar el área para un examen clínico mejor. El examen de los rebordes alveolares puede ser hecho, de forma rápida, buscando posibles fracturas. Las pruebas de sensibilidad y percusión no son confiables en los dientes deciduos debido a los resultados no consistentes. ${ }^{11}$

Como no se puede esperar la cooperación del bebé, las informaciones deben ser obtenidas, en gran parte, por medio de los acompañantes o responsables.

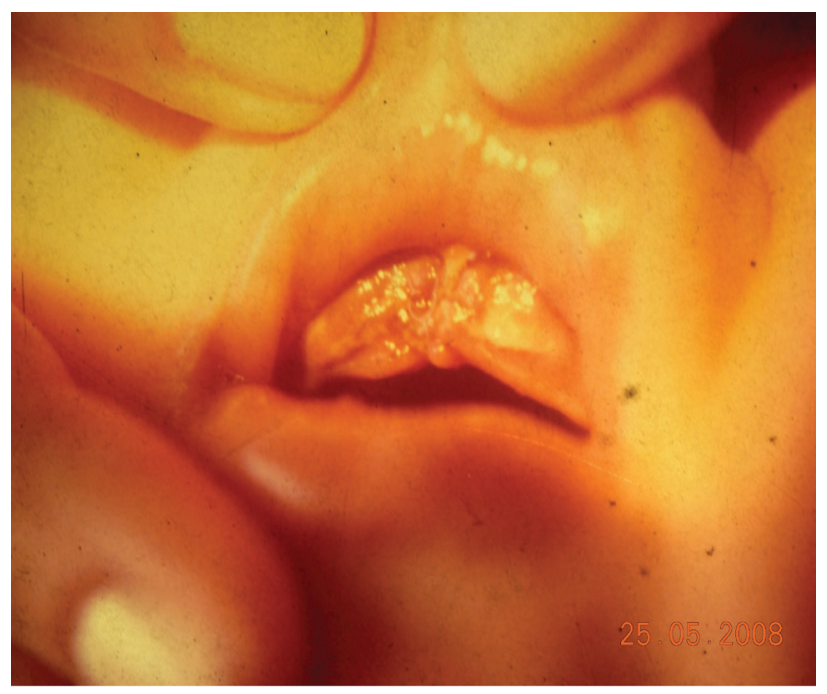

Figura 1. Trauma dental junto con el proceso de erupción. FUENTE: Fotografía tomada por el autor Antonio Nogueira.
Además, será necesario confiar en los exámenes clínicos y observaciones. Examinar, aún, si hubo intrusión, extrusión o avulsión dental. Diferenciar bien si el caso es de intrusión o si es un proceso de erupción del diente aún en progreso, así como si hubo avulsión o intrusión total del diente o si éste aún no ha erupcionado. (Figura 1)

\section{Tratamiento:}

Teóricamente, los tratamientos realizados en casos de traumas bucodentales en cualquier paciente serían los mismos aplicados en los casos ocurridos en bebés. Sin embargo, limitaciones, como la cantidad de dientes en la boca y la madurez psicológica del bebé, hacen que casi siempre no se ejecuten las actitudes terapéuticas deseadas e incluso necesarias.

El número de dientes erupcionados en la arcada es un factor principal cuando se quiere hacer la inmovilización (férula o splint) de algún diente con movilidad o, también, que haya sido recolocado en su lugar de origen. Así, cuantos menos dientes tenga el bebé, más difícil será de hacer la inmovilización, llegando a ser imposible de hacerlo a veces. (Figura 2)

El alisado de borde incisal se hace solamente en situaciones de fractura de esmalte, evitando así grandes trastornos para el niño de edad temprana. En caso de fractura con pérdida de sustancia coronaria, sin exposición pulpar, y en la cual esta pérdida no quede restringida únicamente al esmalte, alcanzando también la dentina (Figura 3), es recomendado que se haga la protección dentinaria.

Recomendamos la aplicación de barniz de Flúor y/o aplicación tópica con solución de Flúor diaria, con la intención de evitar sensibilidad dolorosa. También, en los días iniciales después del trauma, recomendamos alimentación pastosa y libre de cítricos. 


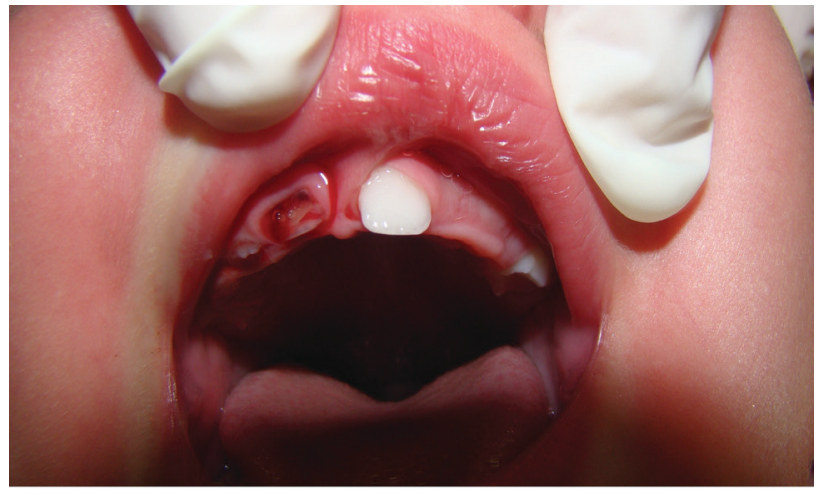

Figura 2. Imposibilidad de inmovilización debido a la insuficiencia de los dientes.FUENTE: Fotografía tomada por el autor Antonio Nogueira.

En casos de fractura donde juzgamos la necesidad de la recomposición coronaria, ésta deberá ser hecha, aunque de forma provisional, pudiendo utilizar el auxilio de las matrices de celuloide en forma de corona, lo que facilita bastante el trabajo a ser hecho. $^{23}$

Las exposiciones pulpares traumáticas de incisivos primarios son raras, pero pueden ser tratadas con Pulpotomía. ${ }^{24}$ En esta condición, el diente debe ser anestesiado inmediatamente, con atención para aplicar la cantidad exacta de anestésico según la determinación de la dosis pediátrica recomendada, a fin de que la pulpotomía sea realizada con el material que mejor se indique.

Para esta intervención, puede ser recomendada la anestesia general como forma de mayor confort para el paciente, preferentemente en ambiente hospitalario, para la seguridad del niño. Si el procedimiento va a ser realizado bajo anestesia local, alertaremos a los padres que el niño podrá agitarse bastante, llorar y gritar, pero que eso no representará necesariamente que el niño esté siendo sometido a dolor o malos tratos. En esta situación, el bebé deberá ser contenido por medio de restricción física, para su seguridad y calidad de la atención.
Los medicamentos como analgésicos, antibióticos, antiinflamatorios y antisépticos bucales (estos aplicados en el lugar del trauma, embebidos en algodón, para que se haga la higienización), podrán ser recomendados, así como se recomienda la vacuna antitetánica o su refuerzo en algunos tipos de traumas ocurridos, como: reimplantes o laceraciones de tejido, entre otros, que causan heridas y tengan origen dudoso y locales sospechosos en cuanto a la higiene.

Consultas sucesivas pueden ser programadas como forma de seguimiento de la evolución del caso. Una nueva intervención, de carácter más definitivo, podrá ser definida, por ejemplo, debido a alguna consecuencia desagradable que pueda surgir, como abscesos, fístulas, movilidades dentales, entre otras.

A su vez, cuando hay desplazamientos parciales, se recomienda el reposicionamiento del diente en el lugar original, suavemente, y su inmovilización, si existe la posibilidad. Cuando no hay esta posibilidad terapéutica y a depender de la gravedad del desplazamiento, la exodoncia puede estar indicada (Figura 4) o, incluso, la no recolocación del diente, pues este última podría causar alteraciones en el germen del diente permanente.

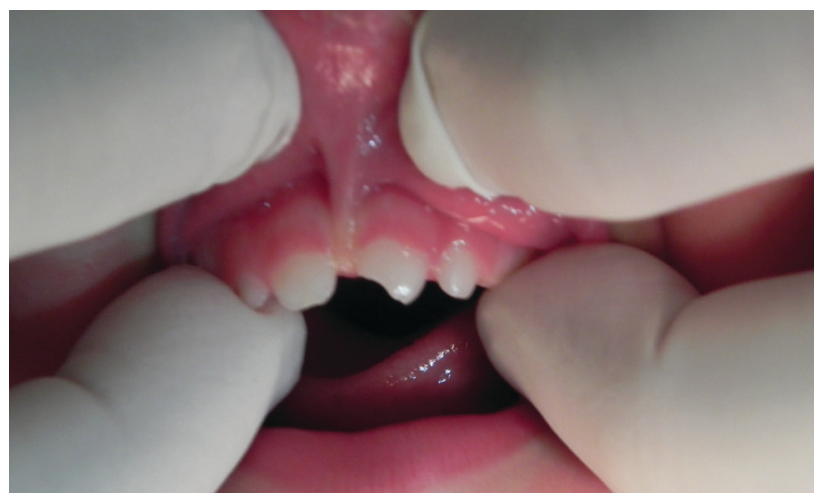

Figura 3. Fractura coronaria con pérdida de sustancia de esmalte y dentina.FUENTE: Fotografía tomada por el autor Antonio Nogueira. 


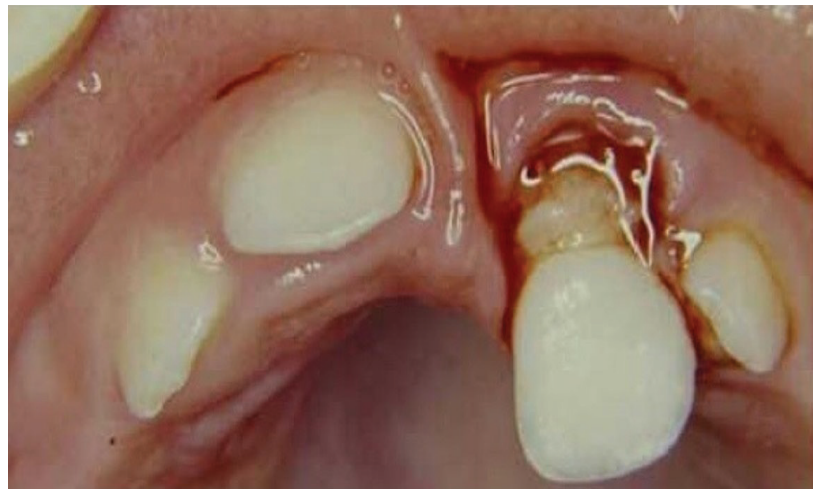

Figura 4. Traumatismo con desplazamiento dental, con indicación para la exodoncia. FUENTE: Fotografía tomada por el autor Antonio Nogueira.

Los casos de pérdida total dental son los que más limitan la decisión terapéutica, pues, por regla general, el niño de edad temprana aún no puede usar una prótesis $\mathrm{y}$, a veces, ni un aparato protésico fijo podrá ser colocado, por la insuficiencia de dientes para anclaje y debido a su madurez psicológica (Figura 5).

Luego, frente a todas esas limitaciones inherentes al niño, es necesario que sea acompañada su evolución y la del caso, para que se tome las

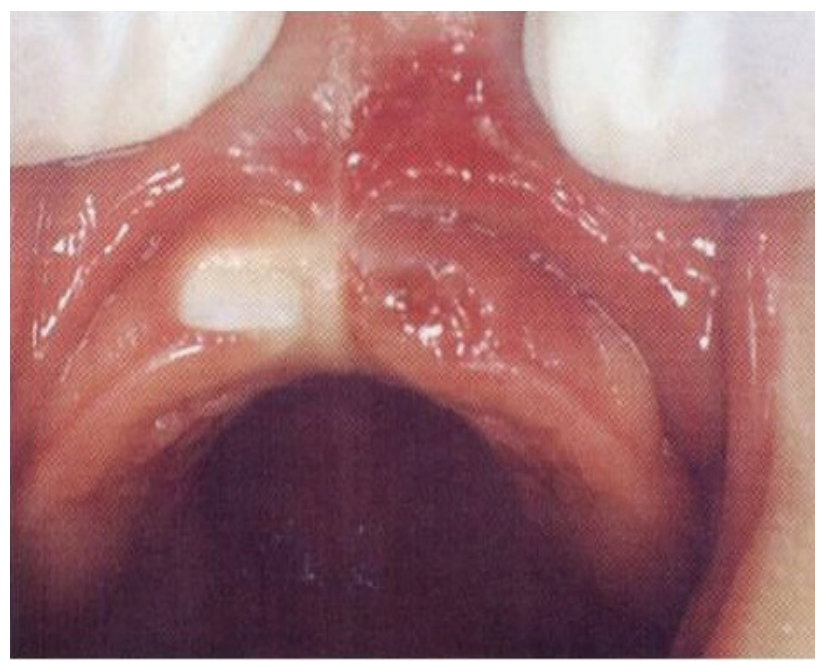

Figura 5. Traumatismo dental, con la imposibilidad de recuperación protésica. FUENTE: Fotografía tomada por el autor Antonio Nogueira. providencias necesarias en los momentos en que se observe la necesidad y claro, tan pronto como sea posible.

Además, hay que estar atento al desarrollo de hábitos de postura lingual, de habla o a una posible asimetría facial $\mathrm{u}$ otro posicionamiento cualquiera que nos haga sospechar que sea un resultado del trauma. Se debe orientar a los padres qué hacer en el momento para disminuir posibles secuelas. No podemos olvidar que el niño en este período de edad temprana está en fase de aprendizaje de todas sus funciones, y los dientes son elementos fundamentales en ese conjunto.

\section{Consecuencias de los traumas dentales:}

La pérdida parcial o total del espacio dental será la consecuencia probable, dependiendo de la edad del niño en el momento del trauma. Con ello, puede ocurrir desviación de línea media, retención dental (figura 6), acortamiento del arco dental haciendo que este paciente necesite de acompañamiento periódico, hasta la decisión de una nueva intervención ortopédica facial e incluso ortodóncica oquirúrgica. ${ }^{25}$

Necrosis o resorciones radiculares internas o externas corono/radiculares, abscesos y fístulas pueden ocurrir, lo que inviabilizará o no la permanencia del diente en la boca. Un diagnóstico bien preciso debe ser hecho y la decisión terapéutica debe ser tomada, ya previendo el futuro oclusal del paciente para su completa recuperación, considerando el sistema estomatognático como un todo. Es importante planificar la solución de los problemas, aunque sea por etapas y considerando cada momento evolutivo del niño.? 


\section{Conclusión}

Queremos con esas informaciones, sobretodo, alertar que los traumas en bebés también necesitan ser apropiadamente evaluados por los Odontopediatras y Cirujanos Dentistas en general. Vale recordar que, las limitaciones en el bebé serán momentáneas, pero las secuelas podrán acompañarle por el resto de su vida. Mutilados o no, abatidos psicológicamente o no, esto dependerá de la inmediatez de las consultas iniciales y acompañamientos sucesivos, hasta el momento en que se cree que es el final del problema instalado. Además, es imprescindible alertar a los responsables en cuanto las fases de tratamiento posibles y las dificultades e imposibilidades. $Y$ con ellos, trabajar juntos, pues forman parte del contexto en que vive el niño, siendo extremadamente necesarios como un apoyo a nuestras acciones, a fin de buscar la recomposición total del sistema estomatognático de sus hijos.

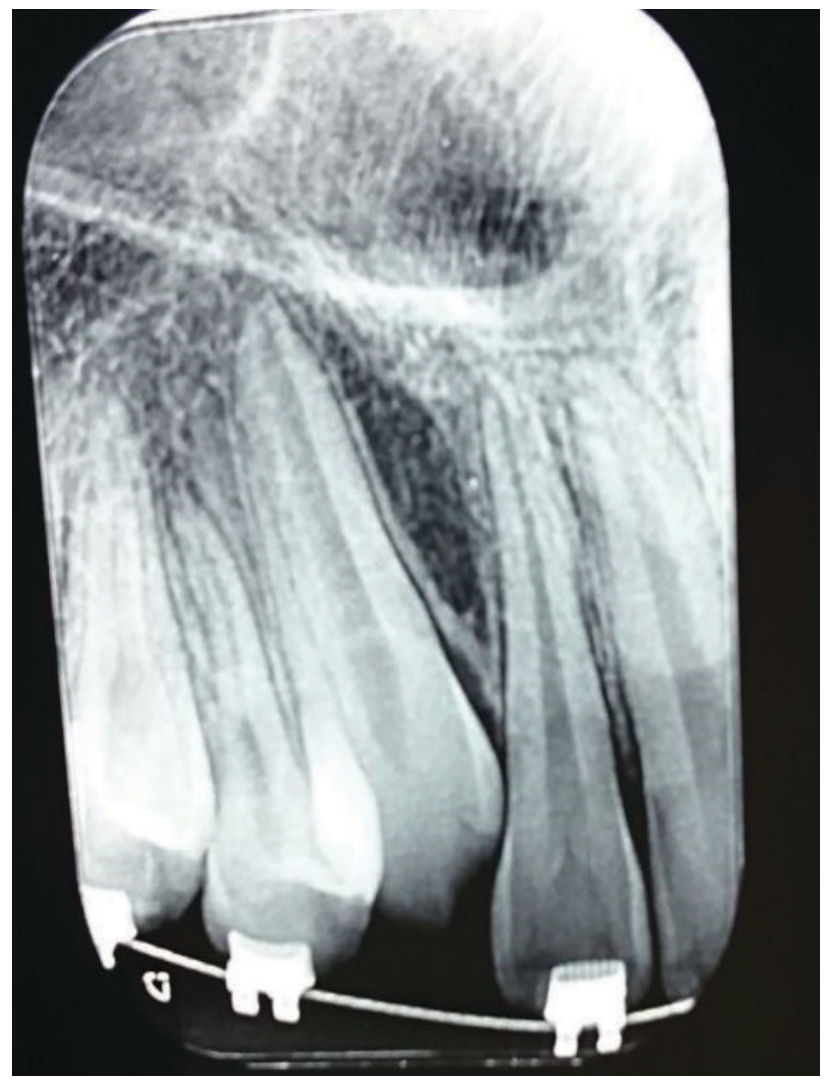

Figura 6. Pérdida dental por traumatismo y consecuentemente disminución de espacio mesio distal para el diente sucesor. FUENTE: Fotografía tomada por el autor Antonio Nogueira.

\section{Referencias bibliográficas}

1- Baldini MH, Lopes CML, Scheidt WA. Prevalência de alterações bucais em crianças atendidas nas clínicas de bebês públicas de Ponta GrossaPr - Brasil.Pesq Odontol Bras. 2001; 15 (4): 302-7.

2- Correa MSNP. Odontopediatria na primeira infância. 3.ed. Santos, São Paulo de 2010.

3- Lam R, Abbott P, Lloyd C, Kruger E, Tennant M. Dental trauma in an Australian rural centre. Dent Traumatol 2008; 24: $663-70$.

4- Avsar A, Topaloglu B. Traumatic tooth injuries to primary teeth of children aged 0-3 years. Dent Traumatol 2009; 25 : 323-7. 5- AssunçãoLRS,Ferelle A. Análise dos traumatismos e suas sequelas na dentição decídua.Pesq Bras Odontop Clin. Int. 2007; 7(02): 173-9.

6- Oliveira AV. Consequências do traumatismo anterior na dentição decídua em crianças atendidas na Bebê Clínica da Unigranrio.[Dissertação de Mestrado] Rio de Janeiro: Universidade do Grande Rio; 2007.

7- Borum MK, Andreasen JO. Sequelae of trauma to primary maxillary incisors. I. Complications in the primary dentition. Endod Dent Traumatol.1998; 14(1): 31-44.

8- Holan G, Ram D. Sequelae and prognosis of intruded primary incisors: a retrospective study. Pediatr Dent. 1999; $21(4)$ : $242-7$. 
9- Cardoso M, Rocha MJC. Federal University of Santa Catarina Traumatized follow-up management routine for traumatized primary teeth- part 1.Dent Traumatol. 2004; 20(6): 307-13.

10- Holan G, Fuks AB. The diagnostic value of coronal dark gray discoloration in primary teeth following traumatic injuries. Pediatr. Dent. 1996; 18(03): $224-7$.

11- Malmgren B, Andreasen JO, Flores MT, Robertson A, DiAngelis AJ, Andersson L, Cavalleri G, Cohenca N, Day P, Hicks ML, Malmgren O, Moule AJ, Onetto J, Tsukiboshi M. International Association of Dental Traumatology guidelines for the management of traumatic dental injuries: 3. Injuries in the primary dentition. Dent Traumatol. 2012; 28(3): $174-82$.

12- ChagasMS, Jácomo DRES, Campos V. Frequência de Hipoplasia do esmalte nos dentes permanentes anteriores, decorrentes de traumatismos nos antecessores. Arquivo Brasil. de Odontologia; 2007.

13- Pugliesi DMC, Cunha RF, Delbem ACB, Sundefeld MLMM. Influence of the type of dental trauma on the pulp vitality and the time elapsed until treatment: a study in patients aged 0-3 years. Dent Traumatol. 2004; 20 (3): 139-42.

14- Cunha RF, Pugliesi DMC, Vieira AEM. Oral trauma in Brazilian patients aged 0-3 years. Dental Tramatol. 2001 ; 17 (5): $210-212$. 15- Zaze ASF, Assunção LRS,Provenzano MGA,Franzin LCS, Ferelle A, Cunha RF. Avaliação de traumatismos dentários em crianças assistidas em um pronto atendimento odontológico. Pesq. Odontol. Bras. 2004; 18 supl: 221.

16- Lombardi SM,Scheller B, Williams B. J.Diagnosis and treatment of dental trauma in a children hospital. PediatrDent. 1998; 20

(2): 112-120.

17- Zembruski-Jaber C, Zambam RC, Condaten V, Cardoso L, Fernandes DSC. Consequências de traumatismos na dentição decídua; Pesq. Bras. de Odontoped. Clinic Integr. 2006; 6 (02): 181- 187.

18- Souza DL, Moreira Neto JJS, Gondim JO, Bezerra Filho JJ. Prevalência de trauma dental em crianças atendidas na Universidade Federal do Ceará; Ver. Odonto Cienc. Ceará: 2008; 23(4): 355-359.

19- Nogueira AJS. Aspectos clínicos dos traumas dentais na 1a․ Infância; Jornal Brasileiro de Odontopediatria e Odontologia do Bebê. 1999; 2 (06): 92 - 95.

20- Granville-Garcia, Menezes VA, Lira PIC. Prevalência e fatores sócio demográficos associados aos traumatismos dentários em pré-escolares; Odontologia Clin. Cient. 2006; 5 (1): 57-64.

21- Della Valle D, Chevitarese ABA, Modesto A, Castro LA de. Frequência de traumatismo dentário em bebês. JBP - Rev Iberoam Odontopediatr Odontol Bebê 2003; 6(34): 464-9.

22- Dourado E, Cypriano RV, Cavalcanti CDS, Domingues AA. Trauma facial em pacientes pediátricos; Revista de Cirurgia e Traumatologia Buco MaxiloFacial. 2004; 4(02); 105-14.

23- Drummond BK. Restoration of primary anterior teeth with composite crowns. N Z Dent J. 1993; 89: 92-5.

24-Kupietzky A, Holan G. Treatment of crown fractures with pulp exposure in primary incisors. Pediatr Dent. 2003; $25: 241-7$.

25- Nogueira AJS. Perdas dentárias precoces e sua importância para as dentições.1ed. Belém-PA: Paka-tatu, 2015.

Recibido: $12 / 02 / 16$

Aceptado: 25/03/17

Correspondencia: Antonio Jose da Silva Nogueira.

Tv. Dom Pedro I, 538, CEP: 66050-100, Belém - Pará. 91 999815540. ajsnog@gmail.com. 